\title{
Structure and Kinematics of Mesoscale Eddies in the Ocean: Theory and Modern Observations
}

\author{
G. K. Korotaev \\ Marine Hydrophysical Institute of RAS, Sevastopol, Russian Federation \\ 凶orotaevgren@mail.ru
}

Purpose. Assessment of general ideas about the character of mesoscale processes in the ocean which came out from the results of the experiment at the POLYMODE polygon, and quantitative verification of the previously developed model of intense eddies from the viewpoint of new data on the eddy characteristics in the open ocean obtained from the satellite altimetry observations constitute the aim of the article.

Methods and Results. Comparison of the characteristic features of mesoscale variability in the ocean resulted from the observational data obtained at the POLYMODE polygon with the generalized analysis of modern satellite altimetry observations showed relevance of the previously formed notions implying that far from the jet currents, the eddies are relatively sparsely distributed over the ocean surface; at that they contain a significant portion of total energy on the mesoscale scales. Quantitative verification of the previously constructed model of an intense mesoscale eddy through its comparison with the eddy features revealed from the satellite altimetry observations showed, overall, satisfactory agreement between the theoretical characteristics and those observed. The altimetry-based median estimate of the eddy structure confirms existence of the eddy core including the main vorticity, and the trap zone in which the particles of the fluid surrounding the core are involved in the orbital motion. According to the observations, the cyclonic eddies drift mainly to the northwest, whereas the anticyclonic ones - to the southwest that is forecasted by the theoretical model. It is shown that the anomalous drift of eddies along the meridian (the cyclonic eddies - to the south and the anticyclonic ones - to the north) is explained by the effect of mean currents. The distances, over which the eddies drift along the meridian, and the inclination angles of the eddy trajectories relative to the parallels are quantitatively close to those observed.

Conclusions. The results of processing the satellite altimetry observations confirm adequacy of the previously developed general notions about the character of the mesoscale processes in the ocean and the physical prerequisites constituting a foundation for the theoretical model of an intense eddy radiating the Rossby waves. At that, interaction of the baroclinic and barotropic modes should be necessarily taken into account for more accurate reproduction of the eddy lifetime.

Keywords: mesoscale eddies, Rossby waves, satellite altimetry, Polygon 70, POLYMODE program

Acknowledgments: the study was carried out in the Marine Hydrophysical Institute of RAS, at financial support of RSF (grant No. 17-77-30001).

For citation: Korotaev, G.K., 2020. Structure and Kinematics of Mesoscale Eddies in the Ocean: Theory and Modern Observations. Physical Oceanography, [e-journal] 27(6), pp. 692-713. doi:10.22449/1573-160X-2020-6-692-713

DOI: $10.22449 / 1573-160 \mathrm{X}-2020-6-692-713$

(C) G. K. Korotaev, 2020

(C) Physical Oceanography, 2020

\section{Introduction}

Fifty years ago, in 1970, Soviet oceanographers brought about a large-scale experiment "Polygon-70". The basic result of the experiment consisted in a detailed documentation of the structure and evolution of a large-scale eddy in the open ocean [1]. Despite the hints made in earlier observations of noticeable variability of various fields in the different parts of the ocean at the scale of tens of days and hundreds of 
kilometers, the experiment "Polygon-70" was the first that gave a complete characteristic of an eddy in the open ocean.

The next stage in studying the mesoscale eddies in the ocean was the USA program MODE (long variant of the experiment title - the Mid-Ocean Dynamics Experiment) [2]. Then, the joint Soviet-American program POLYMODE was planning for detailed study of the ocean mesoscale variability. The name symbolically reflected the contributions of two countries to the studying the mesoscale eddies in the ocean in the experiments "Polygon-70" and MODE. Within the framework of the POLYMODE program in 1977-1978, the hydrological characteristics of marine environment were continuously monitored throughout a year at the $500 \times 500 \mathrm{~km}$ polygon with the resolution corresponding to mesoscale processes. The scientific staffs of the research vessels, which regularly replaced each other at the polygon, measured the seawater temperature and salinity according to the standard scheme [3]. Besides, a network of the moored buoys performed continuous observations of currents at the polygon. The accumulated observations made it possible to describe in sufficient details the features of the ocean mesoscale variability [3, 4].

Discovery and detailed investigation of mesoscale eddies in the ocean gave a powerful incentive to development of oceanology. Having been analyzed, the observations convincingly testified that the current state of the World Ocean was determined by the mesoscale processes and differed significantly from the climatic average. Thereupon, a network of continuous operational observations has been organized in the ocean within the framework of the Global Ocean Observing System (GOOS) program. The main components of this network are buoy profile-meters, the satellite ocean surface temperature and altimetry observations. Satellite altimetry is the basic constituent of the operational oceanic observational system, which allows observing mesoscale variability in all the regions of the World Ocean. Satellite altimetry observations of the ocean have performed since the early 90ies of the last century. The methods for automated processing these observations developed during last 10-15 years make it possible, among other things, to obtain mass estimates of the characteristics of mesoscale eddies [5-7].

In the present study, new data on the characteristics of eddies in the open ocean are used to assess general notions on the mesoscale processes in the ocean resulted from the experiment at the POLYMODE polygon. In the second section, general ideas about the nature of mesoscale processes in the ocean are discussed. In the third section, a new interpretation of the temperature fields' vertical structure at the POLYMODE polygon is given. It shows predominance of the first baroclinic mode in the main thermocline and substantiates further application of the equivalentbarotropic model. The fourth section contains analysis of correspondence of the eddy structure reconstructed using the altimetry measurements to the theoretical concepts. In the fifth section, the eddy motion trajectories are analyzed. The sixth section explains influence of the mean currents upon the eddy displacement direction along the meridian. In the seventh section, the eddy live-times in theory and from the observational data are compared. The eighth section represents general discussion of the obtained results and the additional physical mechanisms that should be taken into account when studying mesoscale eddies. 


\section{General characteristics and statistics of mesoscale eddies in the ocean}

The observational results obtained in the "Polygon-70" experiment became the basis for a discussion about the character of mesoscale variability of the ocean. At that, the introduced concept of a mesoscale vortex was applied to the region, in which at a certain time the rotational motion of fluid is observed. It was proposed to interpret eddies as a superposition of the Rossby waves [8] based on their general movement to the west. However, the observations at the POLYMODE polygon have convincingly shown that the orbital velocity of rotation of liquid particles in the intense eddies considerably exceeds its movement speed [3]. Consequently, the liquid mass rotating around the eddy center is transferred in process of the eddy motion [9]. By now, the concept of intense mesoscale eddies as the formations trapping and transporting the fluid involved in the fluid orbital motion has become generally accepted.

Observations at the POLIMODE polygon also made it evident that the intense eddies are not densely packed. The portion of the ocean surface occupied by the eddies was assessed. It turned out that they occupied about $30 \%$ of the whole area, but contain $80 \%$ of potential energy [9]. Processing of satellite altimetry observations of the ocean surface represented in [6], makes it possible to estimate similar indices on the scale of the entire ocean. According to the data in this paper, 35841 long-lived eddies were observed for 16 years and 2.5 months, therefore, slightly more than 42.6 eddies - per week. Average duration of an eddy lifetime was 32 weeks. Hence, about 1360 eddies were observed simultaneously in the ocean. The eddy mean radius was $90 \mathrm{~km}$. The radius was understood as the distance from the eddy center to the position of the maximum radial velocity [6]. In [9], the eddy radius was defined as the distance to the point where the radial velocity of the liquid particles equals to the speed of the eddy propagation as a whole. This is about $30 \%$ larger than the radius estimated based on the position of the maximum velocity of the orbital motions. Therefore, for comparison, the eddy radius is to be reset equal to about $120 \mathrm{~km}$. Then the part of the ocean surface area occupied by eddies is approximately equal to $1364 \times 3.14 \times 120^{2}=0.62 \times 10^{8} \mathrm{~km}^{2}$. Note that eddies in [6] were observed in the Atlantic, Indian and Pacific oceans. Since the total surface area of these oceans is approximately $3.2 \times 10^{8} \mathrm{~km}^{2}$, we find that eddies occupy about a fifth of it. Taking into account increase of eddies' density when they move from the east coast to the west, this estimate seems to be quite consistent with that at the POLYMODE polygon. Thus, being analyzed, the satellite altimetry observations confirm the proposed in [9] idea of a relatively sparse eddies' distribution over the ocean surface far from the jet currents.

\section{Mode structure of the ocean mesoscale variability}

The equation of the potential eddy conservation serves as a mathematical model for describing mesoscale processes. Since the geostrophic relations are fulfilled with good accuracy on the mesoscales, the potential eddy conservation is described by the equation for the stream function in the horizontal plane. The seawater pressure and, owing to hydrostatics, density are also expressed through the stream function. Finally, the equation of the potential vorticity conservation expressed through the stream function, has the form [4] 


$$
\frac{\partial}{\partial t}\left(\Delta \varphi+\frac{\partial}{\partial z}\left(\frac{f^{2}}{N^{2}} \frac{\partial \varphi}{\partial z}\right)\right)+\frac{\partial\left(\varphi, \Delta \varphi+\frac{\partial}{\partial z}\left(\frac{f^{2}}{N^{2}} \frac{\partial \varphi}{\partial z}\right)\right)}{\partial(x, y)}+\beta \frac{\partial \varphi}{\partial x}=0 .
$$

Here $\varphi$ is the stream function, through which the current velocity components are expressed; $x, y$ are the horizontal coordinates; $z$ is the downward vertical coordinate; $t$ is time; $\Delta$ is the Laplace operator symbol. The second term in equation (1) means the Jacobian. The $X$ and $Y$ coordinate axes are oriented to the east and north, respectively; $N^{2}$ is the Väisälä - Brent frequency; $f$ is the Coriolis parameter; $\beta=\frac{d f}{d y}$. Parameter $\beta$ describes the influence of the spherical Earth. The Väisälä Brent frequency $N$ is expressed using the gravity acceleration $g$, the average density increasing with depth, and the basic density $\rho_{0}=$ const :

$$
N^{2}=\frac{g}{\rho_{0}} \frac{d \bar{\rho}}{d z} \text {. }
$$

When considering the motions of small amplitude, nonlinear terms in the equation (1) can be omitted. At the basin constant depth, the linearized equation (1) is solved by the method of variables separation [4]. The stream function is represented as a product of the function depending on coordinates and time, and the function depending only on the horizontal coordinate $\varphi=\psi(x, y, t) \varphi(z)$. In this case, the function $\varphi(z)$ is found from the solution of the eigenvalues problem

$$
\frac{d}{d z}\left(\frac{f^{2}}{N^{2}} \frac{d \varphi}{d z}\right)=\lambda \varphi
$$

with the boundary conditions $\frac{d \varphi}{d z}=0$ on the surface and bottom of the ocean [4]. Problem (2) has a countable set of eigenfunctions and positive eigenvalues. The barotropic mode corresponding to the eigenfunction that does not change with depth, answers to the zero eigenvalue. The $n$-th baroclinic mode changing the sign $n$ times corresponds to the eigenvalue with number $n$. The eigenvalue has a dimension of the inverse length to the second power. The value $R_{d n}=\lambda_{n}^{-0.5}$ is called the Rossby deformation radius of the $n$-th mode, which depends on the density stratification of marine environment.

In the linear case, each factor in the expansion of the stream function, which depends on the horizontal coordinates and time, is found from the equations of the same type, in which only the value of the Rossby deformation radius changes $R_{d}$ : 


$$
\frac{\partial\left(\Delta \psi-\frac{1}{R_{d}^{2}} \psi\right)}{\partial t}+\beta \frac{\partial \psi}{\partial x}=0 .
$$

If we leave the nonlinear terms in the equation (1), the modes will start to interact with each other. In case a finite number of modes to be considered, one can, using the Galerkin method, derive a finite-dimensional equations system from equation (1). However, it is not clear a priori how many modes are physically significant.

Representation of the equation (1) solution in the form of a projection onto a finite number of modes would greatly simplify physical analysis of the processes taking place in the ocean. Due to this, many studies are aimed at distinguishing the mode composition of the ocean mesoscale variability directly from the observations. The observations at the POLYMODE polygon provided a unique opportunity to analyze the mode composition of the temperature and current velocity fields on the mesoscales.

When studying the mode composition of the mesoscale temperature fluctuations in $[3,10]$, expansion into the empirical orthogonal functions was used. The empirical functions were calculated independently for each of nine hydrological surveys at the POLYMODE polygon performed from July 1977 and June 1978. The analysis of the expansions showed that the first two orthogonal functions describe $80-90 \%$ of dispersion of temperature fluctuations. These functions calculated for each of nine surveys, were similar to each other. Moreover, they almost coincided within the main thermocline differing from each other only in the upper active layer of the ocean. Based on similarity of the vertical profiles of the first two modes outside the active layer, it was concluded that the baroclinic oscillations are of a single-mode character within the main thermocline. However, the calculated empirical functions of the first and the second modes given in $[3,9]$ do not resemble the two lowest dynamic modes corresponding to equation (2). At the same time, the general conclusion on a singlemode character of the baroclinic oscillations on the mesoscale scale within the main thermocline was later on confirmed numerously [11]. Efficiency of the altimetry observations assimilation in the eddy-resolving models of the oceanic circulation is related just to a single-mode character of the baroclinic oscillations on the mesoscale scale. Predominance of one mode provides high correlation between the altimetryderived sea level fluctuations and the seawater density fluctuations in the main thermocline [12].

Let us now dwell on explaining the seeming discrepancy between the empirical functions calculated from the observational data at the POLYMODE polygon, and the dynamic modes. Fig. 1 schematically shows two empirical orthogonal functions averaged over all nine surveys, which consentaneously describe 80-90\% of the temperature fluctuations dispersion at the depths up to $1500 \mathrm{~m}$. At depths below about $250 \mathrm{~m}$, both modes, according to the results in [10], practically coincide. Therefore, in the schematic representation, it was assumed that they took exactly the same values. The first empirical mode (dashed line in Fig. 1) above $250 \mathrm{~m}$ remains positive, reaching its maximum value at the depth $62.5 \mathrm{~m}$. The second mode shown in Fig. 1 as a solid line, changes its sign near the depth $250 \mathrm{~m}$. The local extremum 
of this function near the surface is also located at the depth $62.5 \mathrm{~m}$. Location of the both modes' extremums at the same depth near the surface follows from the results of [10].

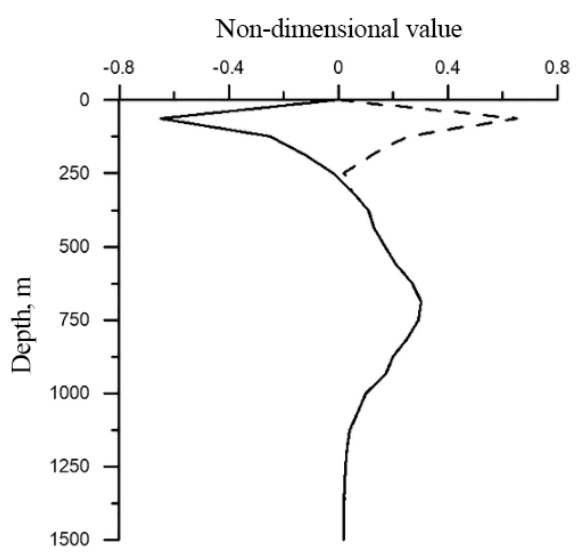

Fig. 1. Schematic representation of two modes of empirical orthogonal functions reproducing 80-90\% of dispersion of temperature fluctuations. In the present scheme, the first mode indicated by the dashed line, coincides with the second one below the $250 \mathrm{~m}$ depth

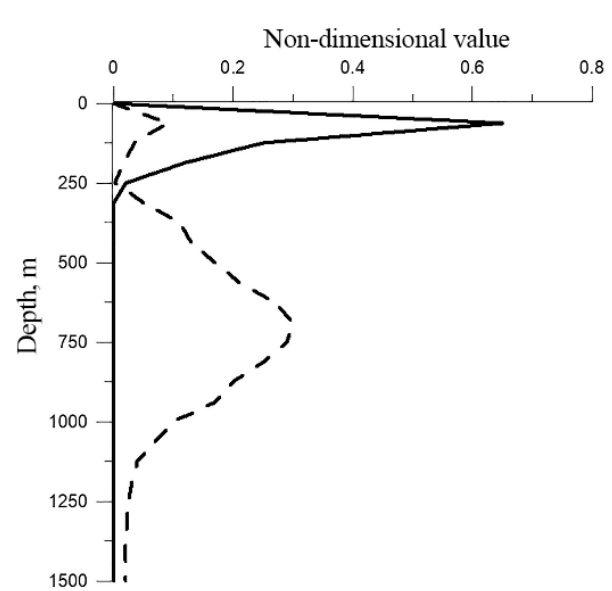

Fig. 2. The eigenfunction of the first baroclinic mode, which is a linear combination of two empirical modes (dashed line), and the concentrated at the surface mode corresponding to the half-difference of the empirical modes (solid line)

A linear combination of the empirical orthogonal modes with specially selected coefficients almost exactly reproduces the profile of the first baroclinic mode estimated from equation (2) with the Neumann conditions on the surface and bottom and the Väisälä - Brent frequency profile calculated using the temperature and salinity average profiles at the POLIMODE polygon (Fig. 2). Semi-difference of the empirical modes reproduces the variability concentrated within the active layer.

The above interpretation of the empirical orthogonal functions (EOF), previously constructed using the data of the hydrological surveys at the POLYMODE polygon, well corresponds to the contemporary ideas about the variability in the seasonal and main thermocline at mesoscales and submesoscales. Indeed, variability on the mesoscals, as noted in [11], is concentrated in the barotropic and the first baroclinic modes. Besides, the numerical simulations [13, 14], satellite observations and small amount of direct observations [15] show that the sub-mesoscale variability in the ocean is concentrated in the upper active layer of the ocean. Thus, the dynamic mode in Fig. 2, which is concentrated close to the surface, can be associated with the submesoscale variability manifestation at the POLYMODE polygon. It is interesting to note that, according to [10], the ratio between the first and the second empirical modes' energies changed during a year. In summer, more energy was contained in the first mode, and in winter - spring, the second mode was more intensive. Such relation between the modes means that in winter - spring, relative significance of temperature fluctuations in the sea near- 
surface layer increases. This behavior probably is related to the sub-mesoscale variability intensification in wintertime [15].

Predominance of the first baroclinic mode permits to simplify significantly studying of kinematics and dynamics of the intense mesoscale eddies. In particular, the equivalent-barotropic model can be used for describing of eddies. The main equation of this model is

$$
\frac{\partial\left(\Delta \psi-\frac{1}{R_{d}^{2}} \psi\right)}{\partial t}+\frac{\partial(\psi, \Delta \psi)}{\partial(x, y)}+\beta \frac{\partial \psi}{\partial x}=0 .
$$

Here, the parameter $R_{d}$ is the Rossby deformation radius of the first baroclinic mode. In the extreme case of small-amplitude motions, equation (4) transforms to equation (3). It describes planetary wave oscillations known as the Rossby waves of the first baroclinic mode. In another extreme case of high-intensity motions with a spatial scale substantially smaller than the Rossby deformation radius, we obtain the equation describing the two-dimensional turbulence [11]. Equation (4) was used in [16] to study structure and kinematics of the intense mesoscale eddies. Further we use the model constructed in this work to compare it with the results of processing satellite altimetry observations [6].

\section{Eddy structure}

The analysis of altimetric observations carried out in [6] permitted to obtain an idea about the internal structure of mesoscale eddies in the ocean. The eddies identified from the altimetry data in this paper were of an almost axisymmetric shape. Thus, each eddy was described by the dependence of the sea level elevation on the distance from the eddy center. Then all the profiles were normalized to the maximum level deviation at the eddy center and to the distance from the center to the maximum velocity of the orbital rotation. After such a normalization, the constructed profiles with a small scatter are concentrated around a certain average profile, being well approximated by the part of parabola. The parabolic profile of sea level, which is a stream function for surface currents, highlights the area of solid-body rotation of fluid particles around the eddy center. With an increase in the distance from the eddy center, the parabolic profile is conjugated with a ring of almost constant value of the level surrounding the eddy [6, Fig. 15, p. 19]. It is curiously that such an approximation of the universal structure of a mesoscale eddy is in good agreement with the eddy model proposed in [16]. It is based on the proposed earlier concept of the equilibrium regime of an intense mesoscale eddy $[9,17,18]$.

In the equilibrium regime, the velocity of the orbital motions of liquid particles in the eddy is assumed to be significantly greater than the phase velocity of its movement. The stream function isoline, which is a separatrix, limits the area of rotation of fluid particles around the eddy center. In [16] it was shown that the eddy of high intensity is almost axisymmetric and its structure is described by the following equation 


$$
\Delta \psi-\frac{1}{R_{d}^{2}} \psi+\beta \bar{y}=\Delta \psi_{0}-\frac{1}{R_{d}^{2}} \psi_{0},
$$

where $\psi_{0}(r)$ is presenting an axisymmetric eddy state at the moment of its formation, $r$ is the distance from the eddy center and $\bar{y}$ is the meridian displacement of the eddy center from its initial position. An axisymmetric solution to equation (5) is found under the boundary conditions

$$
\psi=\frac{\partial \psi}{\partial r}=0 \text { under } r=R
$$

where $R$ - the trap zone radius, where the liquid particles are involved in orbital motion and are transported by the eddy. Three boundary conditions (6) make it possible to find the stream function and the trap zone radius depending on the displacement $\bar{y}$. Since the stream function is proportional to the sea level deviation, the empirical dependence of the sea level on the distance from the eddy center by the part of parabola proposed in [6] is represented as follows

$$
\psi= \begin{cases}-\frac{1}{4} \omega\left(R_{0}^{2}-r^{2}\right)+d, & r \leq R_{0} \\ d, & r>R_{0} .\end{cases}
$$

The expression for $\psi_{0}(r)$ is obtained from (7) when $d=0$. Moreover, from (5) is found that $d=\beta R_{d}^{2} \bar{y}$. Thus, the value of the parameter $d$ determines the distance along the meridian by which the eddy has displaced from the place of formation.

However, the expression (7) is not a solution to problem (5) and (6). The exact solution of (5), (6) includes an axisymmetric solution of the homogeneous equation (5) bounded at the origin of coordinates. Boundary conditions (6) under a finite function $\psi_{0}(r)$ should be slightly modernized. We pose that the stream function vanishes at that position where the orbital velocity of the particles is equal to the phase velocity of long Rossby waves which with a high accuracy corresponding to the eddy velocity. The solution to equation (5) with such boundary conditions is shown in Fig. 3, which should be compared with Fig. 15 from [6]. Good qualitative agreement of the solution of equation (5) with observations gives grounds for further use of the model constructed at [16] for interpreting the results of processing satellite altimetry observations [6]. 


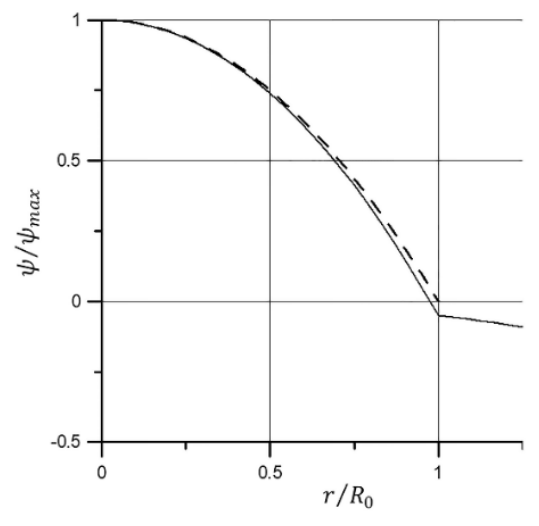

Fig. 3. The stream function profile in the dimensionless coordinates from the solution of (5), if $\psi_{0}(r)$ is preset by the parabolic profile shown by the dashed line

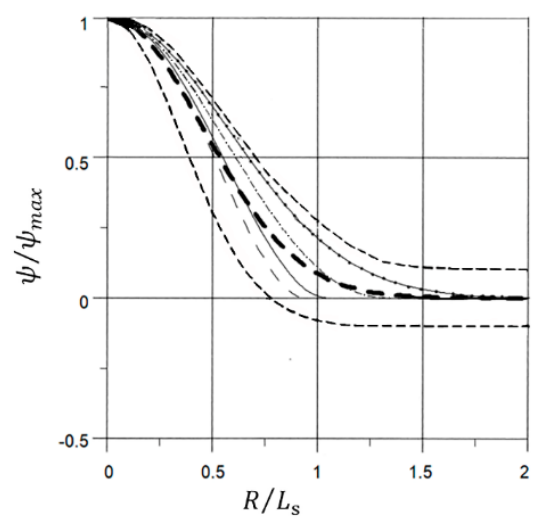

Fig. 4. Normalized stream function profiles at various stages of evolution of the eddy whose radius is twice as much as the Rossby deformation radius. Bold dashed line shows the best approximation of all the Gaussian curve profiles. Thin dashed lines denote dispersion of the observed normalized profiles according to [6]

The Gaussian approximation of the radial distribution of the sea level deviation depending on the distance from the eddy center is also proposed in [6]. It is noted that such an approximation is satisfactory up to a distance from the eddy center equal to $2 / 3$ of its radius. Since the Gaussian approximation is more convenient for analytical calculations, it will be used for further estimates in the present paper.

The solution of equation (5), satisfying the second of the boundary conditions (6), has the following form [6] for any function $\psi_{0}(r)$ :

$$
\psi=\psi_{0}+\beta R_{d}^{2} \bar{y}-\frac{R_{d}}{I_{1}\left(R / R_{d}\right)} \frac{d \psi_{0}(R)}{d R} I_{0}\left(r / R_{d}\right) .
$$

From the first of the boundary conditions (6) follows the relationship between the $R$ radius and the eddy displacement along the meridian obtained in [16]

$$
\bar{y}=\frac{I}{\beta R_{d}} \frac{I_{0}\left(R / R_{d}\right)}{I_{1}\left(R / R_{d}\right)} \frac{d \psi_{0}(R)}{d R}-\frac{\psi_{0}(R)}{\beta R_{d}^{2}} .
$$

It follows from formulas (8) and (9) that the eddy changes its shape during its evolution. The initially Gaussian eddy remains almost Gaussian for large $R$. As $R$ decreases, it deviates more and more from the Gaussian profile. This raises 
the question of whether the Gaussian approximation of the eddy profile will remain satisfactory at all stages of its evolution after the normalization proposed in [6]. Fig. 4 shows the profiles of an initially Gaussian eddy normalized according to [6] at different stages of its evolution. The bold dashed line shows the Gaussian curve, which on average best approximates all eddy profiles at different $R$. Thin dashed lines correspond to the range of profile variability according to [6]. It can be seen that all the calculated profiles are within the scatter range estimated in [6]. Thus, the evolution of the eddy profiles following from the theory fits into the scatter range obtained from the processing of observations.

Formula (9) permits to compare the observed and theoretical displacement of eddies. Calculations using formula (9) were carried out for eddies of different sizes and intensities. The nonlinearity index, in this case equal to the ratio of the maximum velocity of orbital motions to the phase velocity of long baroclinic Rossby waves, in accordance with the estimates given in [6], varied from 2 to 20. Sizes of eddies also varied according to observations presented at [6].

According to formula (9), the maximum value of the eddy displacement along the meridian is achieved at a zero radius of the trap zone. In this case, the eddies can move along the meridian to a distance of up to almost 200 Rossby deformation radii. However, it is clear that the eddy must collapse earlier, when the orbital velocities become sufficiently weak. Therefore, Fig. 5 shows the eddy displacement along the meridian at the moment when the radius of the trap zone becomes equal to the radius where the orbital velocity was maximum at the initial moment i.e., up to $\frac{R_{0}}{\sqrt{2}}$.

As seen from Fig. 5, intense small eddies are displaced along the meridian to the greatest distance. At the same time, large eddies, according to the theory, are displaced along the meridian over relatively short distances.

However, when comparing the results of theory with observations, there is some uncertainty. It is due to the fact that observations of eddies in regions with different values of the Rossby deformation radius in [6] are presented in general statistics. Therefore, Fig. 5 shows the maximum eddy displacement along the meridian from [6], normalized to different values of the Rossby deformation radius. The theoretical estimates in Fig. 5 are shown to be generally consistent with observations. There is even some agreement with the conclusion of [6] that near the equator, where the Rossby radius is large, the nonlinearity parameter does not differ much from the unit. Note, however, that the above theory explains the transport of cyclones (anticyclones) only towards the pole (equator). At the same time, it was noted in [6] that a number of cyclones (anticyclones) are displaced to the equator (pole), which requires a separate consideration. 


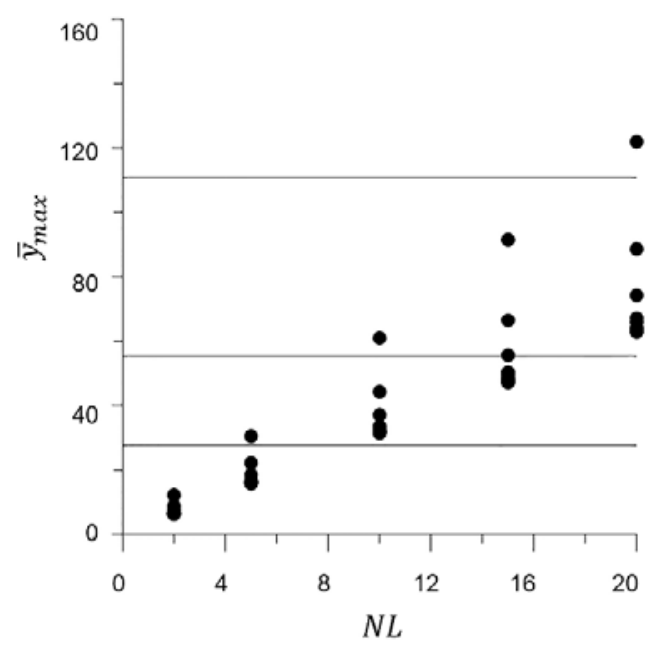

F i g. 5. Maximum meridional displacement of eddies depending on their size and intensity. The nonlinearity parameter value is on the $X$-axis, the eddy maximum displacement along the meridian normalized to the Rossby deformation radius value - on the $Y$-axis. Bold points correspond to 9 eddy sizes from 0.5 to 2.5 of the Rossby deformation radius. The eddy size decreases with distance from the $X$-axis. Horizontal lines denote the eddy maximum displacement along the meridian from [6] normalized to the Rossby deformation radius 20, 40, and $80 \mathrm{~km}$ (from top to bottom)

\section{Eddy movement trajectories}

A model of a moving baroclinic eddy based on the equation (4) which generalized the results obtained in $[9,18]$ is presented in [16]. The system of equations describing the eddy motion includes a quasi-stationary balance of forces acting on it:

$$
\begin{gathered}
\Gamma\left(V-V_{i}\right)=-\frac{V}{R_{d}^{2}} I, \\
-\Gamma\left(U-U_{i}\right)=\left(\beta+\frac{U}{R_{d}^{2}}\right) I .
\end{gathered}
$$

Here $U$ and $V$ are velocity components of the eddy transport; $\Gamma$ is the residual eddy vorticity arising due to the deformation of eddy boundary described by equation (8) caused by its movement; $U_{i}, V_{i}$ are the velocity components induced by Rossby waves radiated by the eddy; $I=-\iint \psi d x d y$, where integration is carried out over the area of the trap zone. The residual vorticity of $\Gamma$ is set by the following expression

$$
\Gamma=4 \pi R \sqrt{\left(U-U_{i}\right)^{2}+\left(V-V_{i}\right)^{2}} .
$$

The velocity induced by Rossby waves is defined by the following equations: 


$$
\begin{gathered}
U_{i}^{\prime}=\frac{\Gamma}{4 \pi} \sqrt{\frac{\beta^{\prime}}{W}\left[2 E\left(\sin \frac{\alpha^{\prime}}{2}\right)-K\left(\sin \frac{\alpha^{\prime}}{2}\right)\right]} \sin \alpha^{\prime}, \\
V_{i}^{\prime}=\frac{\Gamma}{4 \pi} \sqrt{\frac{\beta^{\prime}}{W}\left[2 \cos \alpha \cdot E\left(\sin \frac{\alpha^{\prime}}{2}\right)-\left(1+\cos \alpha^{\prime}\right) K\left(\sin \frac{\alpha^{\prime}}{2}\right)\right]},
\end{gathered}
$$

where $W$ is the eddy movement speed, $\alpha^{\prime}=\alpha-v$, angles $v$ and $\beta^{\prime}$ are defined by the equations below

$$
\beta+\frac{U}{R_{d}^{2}}=\beta^{\prime} \cos v, \quad \frac{V}{R_{d}^{2}}=\beta^{\prime} \sin v,
$$

$U_{i}$ and $V_{i}$ are connected with $U_{i}^{\prime}, V_{i}^{\prime}$ by the following relations

$$
\begin{aligned}
& U_{i}=U_{i}^{\prime} \cdot \cos v-V_{i}^{\prime} \cdot \sin v, \\
& V_{i}=U_{i}^{\prime} \cdot \sin v-V_{i}^{\prime} \cdot \cos v .
\end{aligned}
$$

Equations (10) - (13) permit to find the dependence of the eddy velocity components on the radius of the trap zone. Then, considering relation (9), since $\frac{d \bar{y}}{d t}=V(\bar{y})$, the eddy position is found for each $\bar{y}$.

We will not dwell on the complete calculation of the trajectories of eddy motion in the present paper. We only note that the eddy speed is always slightly less than the phase velocity of long baroclinic Rossby waves of the first mode.

Now we estimate the trajectory inclination angle with respect to latitude, since the variation of this parameter is easy to estimate based on the data given in [6]. Fig. 6 shows the dependences of the trajectory inclination angle of cyclonic eddies on their size and nonlinearity parameter. It can be seen that, as noted earlier, cyclonic eddies, according to the theory, move only to the north. The inclination angles of the trajectories are in a wide range of values from $0^{\circ}$ to about $50^{\circ}$. The inclination angle of the trajectories increases with decreasing eddy radius and decreases with increasing intensity. The maximum angle of inclination of the trajectories of cyclonic eddies according to observations (if only eddies moving to the north are taken into account) is equal to $30^{\circ}$. According to the calculation, the inclination angle of the eddy trajectory exceeds this value only for the case of small eddies of low intensity. Thus, it seems that the theory satisfactorily explains the displacement of cyclonic eddies to the northwest. In this case, the speed of eddies is close, but less than $\beta R_{d}^{2}$. According to observations, the speed of eddies is also close, but slightly higher than the phase velocity of long baroclinic waves of the first mode. At the same time, the theory presented above does not explain the movement of cyclonic eddies to the southwest and gives smaller inclination angles of trajectories in comparison with observations. 


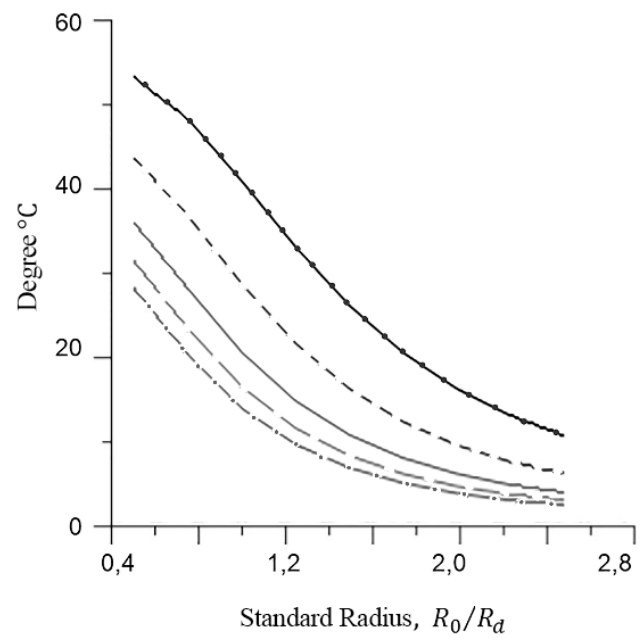

F i g. 6. Inclination angle of the eddy trajectories depending on their size and nonlinearity parameter. The trajectory inclination angle decreases with increase of the nonlinearity parameter from 2 to 20

Based on the results of [16], it can be assumed that the drift of cyclonic eddies to the south and of the anticyclonic ones to the north, is associated with the influence of mean baroclinic currents.

\section{Influence of mean baroclinic currents}

The influence of mean currents on intense eddies, based on the equation (4) is considered below. The mean currents are introduced into the equation (4) by setting $\Psi=-\bar{U} y+\bar{V} x+\varphi$, where $\bar{U}, \bar{V}$ are the components of the mean velocity of currents; $\varphi$ is the stream function describing the eddy. Than for the eddy stream function the following equation is obtained

$$
\frac{d}{d t}\left(\nabla^{2} \varphi-\frac{1}{R_{d}^{2}} \varphi\right)+\frac{\bar{U}\left(\partial \nabla^{2} \varphi\right)}{\partial x}+\frac{\bar{V}\left(\partial \nabla^{2} \varphi\right)}{\partial y}+\frac{\partial\left(\varphi, \nabla^{2} \varphi+\beta y\right)}{\partial(x, y)}=0
$$

Following [16], two new coordinate systems are successively introduced: moving with the velocity of the mean current

$$
x^{\prime}=x-\bar{U} t, \quad y^{\prime}=y-\bar{V} t
$$

and rotated through an angle $\alpha$

$$
x^{\prime \prime}=x^{\prime} \cos \alpha+y^{\prime} \sin \alpha, \quad y^{\prime \prime}=y^{\prime} \cos \alpha-x^{\prime} \sin \alpha .
$$

The angle $\alpha$ is determined by the relations 
where

$$
\bar{U}=\beta^{\prime} R_{d}^{2} \cos \alpha-\beta \cdot R_{d}^{2}, \quad \bar{V}=\beta^{\prime} R_{d}^{2} \sin \alpha,
$$

$$
\beta^{\prime}=\left[\left(1+\frac{\bar{U}}{\beta R_{d}^{2}}\right)^{2}+\left(\frac{\bar{V}}{\beta R_{d}^{2}}\right)^{2}\right]^{1 / 2} \beta .
$$

In the new coordinate system, the equation for the function $\varphi$ almost completely coincides with the equation (4). The difference lies in the appearance of a term $\beta V^{-}$, that is omitted here, assuming that the mean meridional transport is supported by wind stress on the ocean surface. Under this condition, the following expressions for the eddy velocity is obtained

$$
\frac{d \bar{x}^{\prime \prime}}{d t}=-\beta R_{d}^{2}+\tilde{U}, \quad \frac{d \bar{y}^{\prime \prime}}{d t}=\tilde{V},
$$

where $\tilde{U}, \tilde{V}$ are positive and significantly less $\beta^{\prime} R_{d}^{2}$.

Returning to the original coordinate system for the components of the eddy displacement velocity, taking into account (20), (21), the following expressions are obtained:

$$
\begin{gathered}
U=-\beta \cdot R_{d}^{2}+\tilde{U} \cos \alpha-\tilde{V} \sin \alpha, \\
V=\tilde{U} \sin \alpha+\tilde{V} \cos \alpha
\end{gathered}
$$

Thus, in a stationary coordinate system, the eddy continues to move mainly to the west. However, depending on the magnitude $\beta^{\prime}$ and angle $\alpha$, the direction of the eddy movement along meridian changes significantly. A visual representation of the change in the eddy transport along the meridian is given in Fig. 7. There, along the $\mathrm{X}$ and $\mathrm{Y}$ axes, the zonal and meridional projections of the velocity of mean currents, normalized to the velocity of long baroclinic Rossby waves are plotted. The family of circles centered at the point $(-1,0)$ are isolines of the function $\frac{\beta^{\prime}}{\beta}$. The tangent of the angle $\theta$ indicated in Fig. 7 is equal $\tilde{V} / \tilde{U}$ for an eddy of a specific size and intensity. With the selected parameter range, this angle varies from about $15^{\circ}$ to $60^{\circ}$. A straight line drawn at an angle $\theta$ to the $\mathrm{X}$ axis is a boundary, upon crossing which the direction of the meridional drift of cyclonic eddies in the Northern Hemisphere is changed under the influence of the mean flow. If the mean current velocity vector is located below this straight line, then the cyclonic eddy moves to the southwest under the influence of the mean current. The long arrow represents the mean current velocity vector in Fig.7. The dashed arrow represents the phase velocity of long baroclinic Rossby waves $\beta^{\prime} R_{d}^{2}$. 


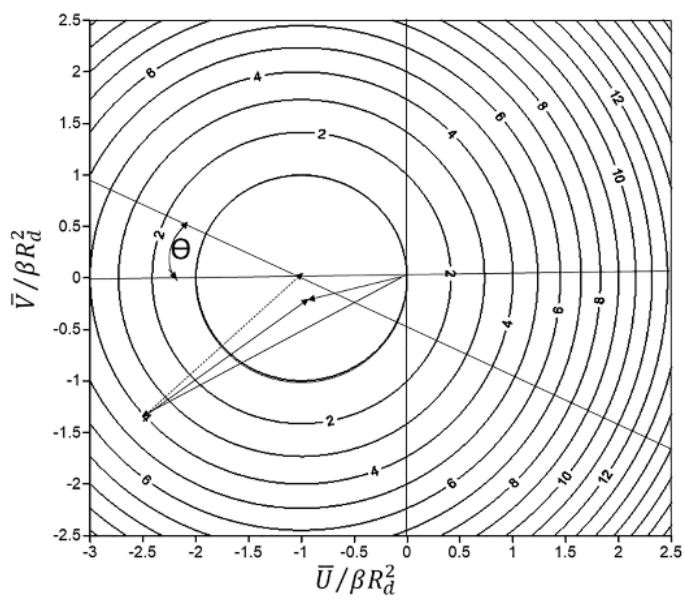

F i g. 7. Circles represent the isolines of parameter $\frac{\beta^{\prime}}{\beta}$ depending on the current zonal (horizontal axis) and meridional (vertical axis) mean velocities, normalized to $\beta^{\prime} R_{d}^{2}$. Strait line separates the directions of the mean currents' velocity vectors, at which the meridional component of the cyclonic eddy drift velocity is positive (upper semi-plane) or negative (lower semi-plane). The solid vector directed to the southwest shows velocity of the mean currents. Dotted vector is the phase velocity of long baroclinic Rossby waves in a moving coordinate system. Solid vector (almost coinciding with it) is the eddy drift velocity in a moving coordinate system. Another solid vector from the origin point is the eddy drift velocity in a fixed coordinate system

It follows from the theory presented in Section 5 that an eddy in a moving coordinate system moves with a velocity whose vector is slightly rotated to the right of the dotted vector. The length of the rotated vector is slightly less than that of the dotted vector. The vector sum of the velocities of the mean current and the eddy movement in the moving coordinate system gives the direction of the eddy propagation in the absolute coordinate system. Fig.7 clearly demonstrates that the meridional component of the cyclonic eddy propagation velocity is directed to the south. Consequently, mean currents can change the direction of eddies drift along the meridian. The explanation for this effect is quite simple. The pycnocline slope associated with the mean flow affects the eddy motion in the same way as the $\beta$ effect [16]. When an eddy moves on the f-plane, the cyclonic eddy moves in the direction opposite to the pycnocline depth gradient. Taking into account the dependence of the Coriolis parameter on latitude, the cyclonic eddy moves in the direction of the gradient $\frac{f}{h}$, where $h$ is the space dependent depth of the pycnocline.

\section{Eddy lifetime}

One of the major results of [6] was the estimation of the lifetime of intense mesoscale eddies. As it turned out, eddies can be rather long-lived formations. In total, from 1992 to 2008, 620 eddies were identified in [6], which were observed for more than two years. It is of interest to compare the estimate of the eddy lifetime 
according to the model with observations. As in Section 4, the eddy lifetime will be assumed to be the moment when the radius of the trap zone reaches the value $\frac{R_{0}}{\sqrt{2}}$.

Table 1 shows theoretical estimations of the eddy lifetime in dimensionless variables for different values of the eddy radius normalized to the Rossby deformation radius and nonlinearity parameter. The time scale of the normalizing vortex lifetime is $\beta^{-1} R_{d}^{-1}$. Eddies in the range of $10-60^{\circ}$ latitude were analyzed in [6]. This latitude range corresponds to a Rossby deformation radius within 20$110 \mathrm{~km}$. Accordingly, it is found that the dimensionless value of the eddy lifetime according to observations depending on the value of the Rossby deformation radius, varies from about 20 to 140 . Those values of the theoretical estimate of eddy lifetime which are less than 140 are highlighted in Table 1 . It can be seen that the theoretical estimates of the eddy lifetime for values of the nonlinearity parameter more than 10 significantly exceed those obtained from observations.

Table 1

Theoretical estimates the eddies' lifetime

\begin{tabular}{c|c|c|c|c|c}
\hline \multirow{2}{*}{$R_{0}$} & \multicolumn{5}{c}{$N L$} \\
\cline { 2 - 6 } & 2 & 5 & 10 & 15 & 20 \\
\hline 0.50 & 61.15 & 138.31 & 276.16 & 429.29 & 597.84 \\
0.75 & 31.51 & 82.37 & 189.24 & 321.73 & 478.18 \\
1.00 & 23.69 & 72.23 & 186.70 & 338.39 & 524.62 \\
1.25 & 22.44 & 77.64 & 217.28 & 409.33 & 650.12 \\
1.50 & 24.39 & 92.24 & 271.01 & 522.17 & 840.88 \\
1.75 & 28.57 & 114.47 & 346.41 & 676,53 & 1098.59 \\
2.00 & 34.70 & 144.20 & 444.54 & 875.65 & 1429.63 \\
2.25 & 42.73 & 181.84 & 567.40 & 1124.16 & 1842.18 \\
2.50 & 52.74 & 228.00 & 717.41 & 1427.26 & 2345.25 \\
\hline
\end{tabular}

As noted before, there are certain difficulties in comparing the calculations with observations, since in the analysis of observations, eddies from areas with different values of the Rossby deformation radius were included in the general statistics. Nevertheless, based on the results presented in [6], the reasons for the most significant differences between the theoretical estimates of the eddy lifetime and the observed ones can be clarified.

A histogram of size and amplitude distribution of the observed eddies adapted from [6] is given in Fig. 8. The horizontal axis represents the sea level deviation $\alpha$ at the center of eddy. The eddy radius $L_{s}$ is plotted along the vertical axis. It is 
estimated in [6], that $R_{0}=0,64 L_{s}$ if $R_{0}$ is the radius of a Gaussian function that approximates an eddy of $L_{s}$ size. The known relationship between the stream function and the sea surface level deviation

$$
\psi=\frac{g}{f} \varsigma
$$

provide the possibility to express the values of nonlinearity parameter $N L$ through the maximum sea level deviation $\alpha$ in eddy center:

$$
N L=\frac{V_{\max }}{\beta R_{d}^{2}}=\frac{\psi_{\max }}{\beta R_{d}^{2} R_{0}} \sqrt{\frac{e}{2}} \approx 0,75 \frac{g}{f \beta R_{d}^{2}} \cdot \frac{\alpha}{L_{s}} .
$$

Thus, for a predetermined Rossby deformation radius, the nonlinearity parameter $N L$ will be constant along any straight line passing through the origin in the histogram in Fig. 8. Two such lines are presented in Fig. 8. One cuts off the region of scales and amplitudes at which eddies were absent in the observations. The second straight line corresponds to $N L=1$ at a Rossby deformation radius of $40 \mathrm{~km}$. Note that on the lower straight line with a Rossby deformation radius of $40 \mathrm{~km}$, the nonlinearity parameter is approximately equal to 8 . As the deformation radius increases, the nonlinearity parameter along the lower straight line will decrease, and the line on which $N L=1$ will rotate clockwise. Consequently, eddies at the equator have a small degree of nonlinearity. With a decrease in the Rossby radius on the lower straight line, the nonlinearity parameter will increase. The line on which $N L=1$ will rotate counterclockwise. Consequently, according to the proposed histogram interpretation, the most nonlinear eddies should be located at high latitudes. This conclusion corresponds to Fig. 17 from work [6, p. 22]. In particular, at $R_{d}=40 \mathrm{~km}$, the nonlinearity parameter of the observed eddies is less than eight. Accordingly, overestimated values of the eddy lifetime in the columns of Table 1 with a nonlinearity parameter of 10-20 do not refer to actually observed eddies for the latitude interval where $R_{d}=40 \mathrm{~km}$. It can also be noted that, according to Fig. 8, large eddies are absent in observations at higher amplitudes. Accordingly, in Table 1 the maximum eddy lifetime values (located in the lower right corner) should be discarded as not presented in observations.

However, even after such remarks, theoretical estimates of the eddy maximum lifetime remain overestimated by two - four times. It can be concluded from this analysis that the theory underestimates the energy losses of eddies during their evolution. The average baroclinic currents can partially correct the lifetime. Indeed, according to the results of the previous section, the damping of eddies in the presence of mean currents is determined by the modified beta effect. Energy flow to baroclinic waves, according to Fig. 7, can increase several times with the corresponding direction of the mean currents. Accordingly, the eddy lifetime will be reduced. Another possible and, as it seems, the most significant is the radiation of barotropic Rossby waves by the baroclinic eddy. The mechanism of barotropic Rossby waves radiation is described in $[16,19]$, but only for infinitely deep ocean. Unfortunately, it is difficult to generalize the results obtained in these works to more realistic 708

PHYSICAL OCEANOGRAPHY VOL. 27 ISS. 6 (2020) 
conditions. The main cause is that the emitted barotropic waves have a wavenumber close to $R_{d}^{-1}$. Therefore, finite-amplitude barotropic Rossby waves will affect the characteristics of the eddy trap zone. Note that the eddy lifetime is inversely proportional to the meridional velocity component its propagation. At the same time, the energy flux from the eddy to the waves is approximately direct proportional to that velocity component. Thus, the reduction in the eddy lifetime in comparison with the estimates given in Table 1, up to the observed two years requires an energy flux to barotropic waves equal to or exceeding the flux to baroclinic waves.

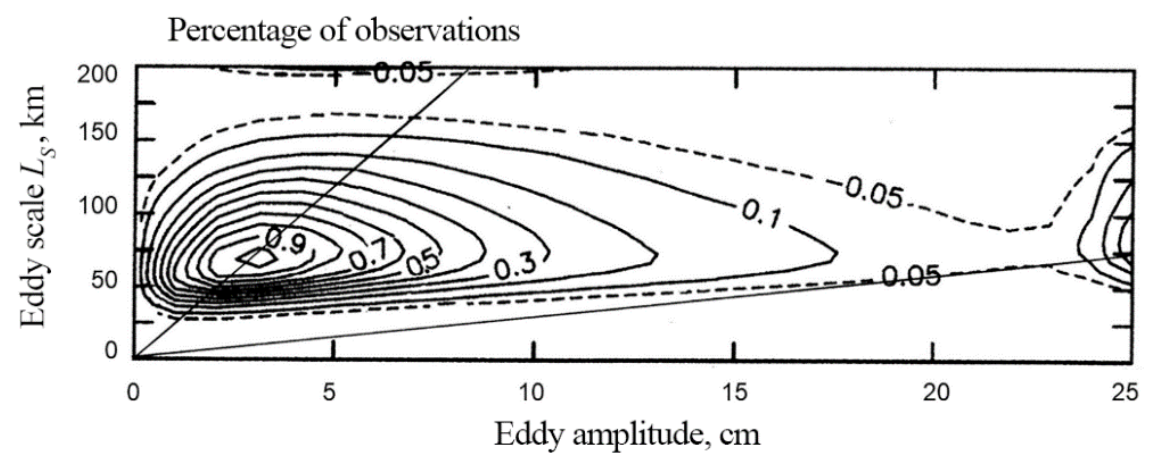

F i g. 8. Histogram of the observed eddies distributed based on their sizes and amplitudes according to [6]. Two straight lines are plotted on the histogram. Below the lower straight line, there is an area of scales and amplitudes at which no eddies were observed. The upper straight line corresponds to $N L=$ 1 with the Rossby deformation radius equal to $40 \mathrm{~km}$

The results of [6] also provide possibility of more accurate assessment of the correspondence of the theoretical estimate of the maximum eddy lifetime to observations. Let us consider the trajectories of eddies with a lifetime of more than two years shown in Fig. 4, $f$ [6, p. 11]. As it turns out, long-lived eddies are located mainly in the areas of trade currents. For a theoretical estimate of the eddy lifetime at these latitudes, we take the Rossby radius equal to $40 \mathrm{~km}$, and the upper nonlinearity boundary in accordance with Fig. 8 is set equal to 7.81. Calculations of the eddy lifetime are given in Table 2 . The cells, in which, according to the histogram shown in Fig. 8, eddies of the corresponding size and degree of nonlinearity are absent in the observations are marked in gray. Note also that the first row of Table 2 corresponds to eddies that are not resolved by altimetry observations due to their small size. The cells in which the dimensionless eddies lifetime is less than two years are highlighted in light green. From Table 2 it can be seen that the theoretical estimates for some eddies still exceed two years by up to 2.5 times. Note that in the trajectories of eddies in Fig. 4, $f$ from [6], an anomalous movement of cyclonic eddies to the southwest is observed. This behavior corresponds to the results of the previous section, since the considered eddies are in the zone of trade currents. As noted above, under effect of currents the lifetime of eddies, given in Table 2, can be reduced. An additional source of a more rapid damping of eddies can also be the radiation of barotropic Rossby waves discussed above, which is not taken into account in the considered theoretical model. 
Theoretical estimates of the eddies' lifetime at the deformation radius equal to $40 \mathbf{~ k m}$

\begin{tabular}{c|c|c|c|c|c}
\hline \multirow{2}{*}{$R_{0}$} & \multicolumn{5}{|c}{$N L$} \\
\cline { 2 - 6 } & 1.5 & 2.25 & 3.68 & \multicolumn{1}{c}{6.06} & 7.81 \\
\hline 0.75 & 23.98399 & 35.36973 & 58.73100 & 102.7773 & 139.1543 \\
1.00 & 17.27249 & 27.08840 & 48.84097 & 93.1768 & 131.7472 \\
1.25 & 15.69198 & 26.10093 & 50.43302 & 102.5631 & 149.3385 \\
1.50 & 16.49537 & 28.74468 & 58.34838 & 123.6742 & 183.3549 \\
1.75 & 18.87022 & 33.97086 & 71.22154 & 154.8959 & 232.1539 \\
2.00 & 22.55438 & 41.48980 & 88.79558 & 196.2503 & 296.1492 \\
2.25 & 27.48885 & 51.28602 & 111.22720 & 248.3994 & 376.5113 \\
2.50 & 33.69849 & 63.46043 & 138.84310 & 312.2513 & 474.7460 \\
\hline
\end{tabular}

\section{Discussion}

The content of the previous sections of this paper allows us to conclude that the ideas about the nature of the ocean mesoscale variability developed based on the analysis of the POLYMODE test site hydrological surveys are confirmed by new observations. The processing of satellite altimetry observations carried out in [6] shows that far from the jet currents, the main energy of the ocean mesoscale variability is contained in high-intense eddies. Open ocean eddies are long-lived nonlinear formations that capture and transport significant volumes of fluid over long distances. The space between eddies are filled with background structures of much lower intensity, which rapidly change their shape [6] and, most likely, have a wave nature. Despite the high fraction of the energy content in eddies, satellite observations confirm that they are located relatively rarely on the ocean surface and can be considered as isolated structures. This circumstance served as the basis for expanding theoretical studies of the evolution of intense isolated eddies in the ocean.

The model of an intense isolated eddy, developed within the framework of research under the POLYMODE program $[9,16]$, is used here for comparison with the satellite observations generalized in [6]. The assessment of the quality of this model given above showed overall a satisfactory agreement of the theoretical characteristics with the observed ones, despite significant simplifications. The median estimate of the eddy structure given in [6] confirms the existence of the eddy core, in which the main vorticity is concentrated, and the trap zone, in which particles of the fluid surrounding the core are involved in orbital motion. Cyclonic eddies, according to observations, mainly move to the northwest, and anticyclonic ones - to the southwest, as prescribed by the theoretical model. The distances over which the eddies move along the meridian, the angles of inclination of the eddy trajectories relative to the parallels are close to the observed ones.

A slight generalization of the theoretical model makes it possible to include in consideration mean currents. This generalization provides the explanation of seemingly contradiction to the theory of cyclonic eddies movement southwestwards 
(and, accordingly, anticyclonic eddies northeastwards) in certain regions of the ocean.

Note also that small-scale fluctuations on the trajectories of eddy motion constructed from the data of altimetric satellite observations are often observed. Such oscillations can be associated with the interaction of eddies with Rossby waves. In works $[16,18]$ it is shown that eddies not only radiate waves, but also, under certain conditions, can receive energy from the waves. With an increase in energy, the cyclonic eddy begins to move along the meridian to the south. In this case, oscillations will appear on the smooth eddy trajectory. In addition, eddies located at a relatively small distance from each other can mutually disturb the trajectories of their motion. Moreover, as shown by numerical simulations [20], when the eddies of the same sign approach each other, they can merge, which is typical for twodimensional turbulence [21].

The largest discrepancy between theory and observations is associated with the estimate of the eddy lifetime. Eddies should live much longer according to the theory than in reality. Note that the theoretical eddy lifetime depends on the meridional velocity component of its movement. At the same time, the distance to which the eddy should move is estimated quite accurately. Therefore, the theoretical estimate of the lifetime is sensitive to the accuracy of estimating the velocity of the eddy displacement along the meridian. The studies of the socalled beta gyre, presented in [22], should show how consistent are the estimates of this velocity, obtained from calculating the field of waves emitted by eddies.

However, most likely, the refinement of the meridional component of the eddy displacement velocity within the framework of the equivalent barotropic model (4) will not explain such a significant discrepancy between theory and observations. As noted in Section 7, the most significant drawback of model (4), which causes the discrepancy between theoretical and observed estimates of the eddy lifetime, is the exclusion from the consideration of the barotropic mode. In reality, during its motion the eddy will radiate Rossby waves of both the first baroclinic and barotropic modes. In this case, the energy loss by the eddy for wave radiation will increase, and, accordingly, its lifetime will decrease. Thus, in order to achieve a better quantitative correspondence of the eddy characteristics in the model to observations, one should take into account the radiation of the first baroclinic and barotropic modes. This means a transition from the equivalent barotropic model (4) to the two-layer fluid model.

The transition to a simultaneous description of the barotropic and the first baroclinic modes in the study of the dynamics of intense eddies is also important for understanding the processes of their generation. Indeed, within the framework of equation (4), only the evolution of the open ocean eddies can be described. Observations seem to leave such a possibility, since it is noted in [6] that a significant proportion of the observed eddies are formed because of meandering of the eastern boundary currents. Having originated at the eastern boundaries of the ocean, such eddies must then move westward, filling open areas of the ocean. However, such a simple scheme is not consistent with observations. As noted in [6], according to observations, eddies are also formed in the middle of the oceans. In this case, the most probable reason of their origin is considered to be the baroclinic instability of mean currents, for the description of which it is necessary to take into account the 
interaction of the baroclinic and barotropic modes of motion. As the results of [23] show, mean currents can also feed eddies with energy during their evolution. This process also requires taking into account both baroclinic and barotropic modes of motion. It is quite probable that studies of the dynamics of isolated eddies in a twolayer fluid will provide a better understanding of development of geostrophic turbulence on a beta plane, including the redistribution of energy between the baroclinic and barotropic modes of motion noted by Rhines [24].

\section{REFERENCES}

1. Brekhovskikh, L.M., Ivanov-Frantskevich, G.N., Koshlyakov, M.N., Fedorov, K.N., Fomin, L.M. and Yampolsky, A.D., 1971. Some Results of the Hydrophysical Experiment on the "Polygon" in the Tropical Atlantic. Izvestiya Akademii Nauk SSSR. Fizika Atmosfery i Okeana, 7(5), pp. 511-527 (in Russian).

2. The MODE Group, 1978. The Mid-Ocean Dynamics Experiment. Deep Sea Research, 25(10), pp. 859-910. https://doi.org/10.1016/0146-6291(78)90632-X

3. Nelepo, B.A., Bulgakov, N.P., Timchenko, I.E., Korotaev, G.K., Aretinskiy, G.Yu., Babiy, M.V., Ganson, P.P., Golubev, Yu.N., Grishin, G.A. [et al.], 1980. [Mesoscale Eddies in the Ocean]. Kiev: Naukova Dumka, 288 p. (in Russian).

4. Kamenkovich, V.M., Koshlyakov, M.N. and Monin, A.S., 1986. Mesoscaleal Eddies in the Ocean. Dordrecht: Springer, 376 p. https://doi.org/10.1007/978-94-009-4502-9

5. Greenslade, D.J.M., Chelton, D.B. and Schlax, M.G., 1997. The Midlatitude Resolution Capability of Sea Level Fields Constructed from Single and Multiple Satellite Altimeter Datasets. Journal of Atmospheric and Oceanic Technology, 14, pp. 849-870. https://doi.org/10.1175/1520-0426(1997)014<0849:TMRCOS>2.0.CO;2

6. Chelton, D.B., Schlax, M.G. and Samelson, R.M., 2011. Global Observations of Nonlinear Mesoscale Eddies. Progress in Oceanography, 91(2), pp. 167-216. doi:10.1016/j.pocean.2011.01.002

7. Chen, G., Han, G. and Yang, X., 2019. On the Intrinsic Shape of Oceanic Eddies Derived from Satellite Altimetry. Remote Sensing of Environment, $228 . \quad$ pp. 75-89. https://doi.org/10.1016/j.rse.2019.04.011

8. McWilliams, J.C. and Robinson, A.R., 1974. A Wave Analysis of the Polygon Array in the Tropical Atlantic. Deep Sea Research and Oceanographic Abstracts, 21(5), pp. 359-368. https://doi.org/10.1016/0011-7471(74)90040-0

9. Korotaev, G.K., 1980. Structure, Dynamics and Energetic of Mesoscale Variability of the Ocean. Preprint. Sevastopol: Marine Hydrophysical Institute, 64 p. (in Russian).

10. Korotaev, G.K., Kosnyrev, V.K. and Shevchenko, E.A., 1979. The Structure of Disturbances in the Temperature Field of Mesoscale Eddy Formations. In: MHI, 1979. Morskie Gidrograficheskie Issledovaniya [Marine Hydrophysical Research], Sevastopol: MHI. Issue 2, pp. 167-174 (in Russian).

11. Ferrari, R. and Wunsch, C., 2009. Ocean Circulation Kinetic Energy: Reservoirs, Sources, and Sinks. Annual Review of Fluid Mechanics, 41, pp. 253-282. https://doi.org/10.1146/annurev.fluid.40.111406.102139

12. Korotaev, G.K., 2010. Baroclinic Layer of the Sea: from Lineikin's Theory to Operational Oceanography. In: A. V. Frolov and Yu. D. Resnyansky, Eds., 2010. Modern Problems of Ocean and Atmosphere Dynamics: The Pavel S. Lineykin Memorial Volume. Moscow: Triada, pp. 79-98 (in Russian).

13. Lapeyre, G. and Klein, P., 2006. Dynamics of the Upper Oceanic Layers in Terms of Surface Quasigeostrophy Theory. Journal of Physical Oceanography, 36(2), pp. 165-176. doi:10.1175/JPO2840.1 
14. Ponte, A.L. and Klein, P., 2013. Reconstruction of the Upper Ocean 3D Dynamics from HighResolution Sea Surface Height. Ocean Dynamics, 63, pp. 777-791. doi:10.1007/s10236-0130611-7

15. Callies, J., Ferrari, R., Klymak, J. and Gula, J., 2015. Seasonality in Submesoscale Turbulence. Nature Communications, 6, 6862. https://doi.org/10.1038/ncomms7862

16. Korotaev, G.K., 1997. Radiating Vortices in Geophysical Fluid Dynamics. Surveys in Geophysics, 18, pp. 567-618. https://doi.org/10.1023/A:1006583017505

17. Korotaev, G.K., 1988. Theoretical Modeling of the Ocean Mesoscale Variability. Kiev: Naukova Dumka, 160 p. (in Russian).

18. Korotaev, G.K., and Fedotov, A.B., 1994. Dynamics of an Isolated Barotropic Eddy on a BetaPlane. Journal of Fluid Mechanics, 264, pp. 277-301. https://doi.org/10.1017/S0022112094000662

19. Flierl, G.R., 1984. Rossby Wave Radiation from a Strongly Nonlinear Warm Eddy. Journal of Physical Oceanography, 14(1), pp. 47-58. https://doi.org/10.1175/15200485(1984)014<0047:RWRFAS>2.0.CO;2

20. Korotaev, G.K. and Dorofeev, V.L., 1999. Evolution of an Ensemble of Geostrophic Vortices on a $\beta$-plane. Izvestiya, Atmospheric and Oceanic Physics, 35(2), pp. 182187.

21. McWilliams, J.C., 1984. The Emergence of Isolated Coherent Vortices in Turbulent Flow. Journal of Fluid Mechanics, 14 pp. 21-43. https://doi.org/10.1017/S0022112084001750

22. Reznik, G.M. and Kravtsov, S.V., 2020. Singular Vortices on a Beta-Plane: A Brief Review and Recent Results. Physical Oceanography, 27(6), pp. 659-676. doi:10.22449/1573-160X2020-6-659-676

23. Sutyrin, G.G., 2020. How Oceanic Vortices Can Be Super Long-Lived. Physical Oceanography, 27(6), pp. 677-691. doi:10.22449/1573-160X-2020-6-677-691

24. Rhines, P.B., 1979. Geostrophic Turbulence. Annual Review of Fluid Mechanics, 11, pp. 401441. https://doi.org/10.1146/annurev.fl.11.010179.002153

About an author:

Gennadiy K. Korotaev, Scientific Director, Marine Hydrophysical Institute of RAS (2 Kapitanskaya str., 299011, Sevastopol, Russian Federation), Corresponding member of RAS, Dr. Sci. (Phys.-Math.), Professor, ResearcherID: K-3408-2017, gkorotaev@gmail.com

The author has read and approved the final manuscript.

The author declares that he has no conflict of interest. 